\title{
All-Fabric-Based Flexible Capacitive Sensors with Pressure Detection and Non-Contact Instruction Capability
}

\author{
Xiaorui Ye ${ }^{1}$, Mingwei Tian ${ }^{1,2, *}$, Ming Li $^{1}$, Hang Wang ${ }^{1,2, *}$ and Yangcheng Shi ${ }^{3}$ \\ 1 Research Center for Intelligent and Wearable Technology, College of Textiles \& Clothing, Qingdao University, \\ Qingdao 266071, China; yexiaorui19@126.com (X.Y.); lm9609@163.com (M.L.) \\ 2 Research Center for Intelligent and Wearable Technology, College of Textiles and Clothing, \\ State Key Laboratory of Bio-Fibers and Eco-Textiles, Collaborative Innovation Center for Eco-Textiles of \\ Shandong Province and the Ministry of Education, Intelligent Wearable Engineering Research Center of \\ Qingdao, Qingdao University, Qingdao 266071, China \\ 3 Anhui Disheng Weaving \& Finishing Co., Ltd., Bozhou 233600, China; shiyangcheng@163.com \\ * Correspondence: mwtian@qdu.edu.cn (M.T.); wanghang@qdu.edu.cn (H.W.)
}

check for updates

Citation: Ye, X.; Tian, M.; Li, M.; Wang, H.; Shi, Y. All-Fabric-Based Flexible Capacitive Sensors with Pressure Detection and Non-Contact Instruction Capability. Coatings 2022, 12, 302. https://doi.org/10.3390/ coatings 12030302

Academic Editor: Fabien Salaün

Received: 17 January 2022

Accepted: 10 February 2022

Published: 23 February 2022

Publisher's Note: MDPI stays neutral with regard to jurisdictional claims in published maps and institutional affiliations.

Copyright: (C) 2022 by the authors. Licensee MDPI, Basel, Switzerland. This article is an open access article distributed under the terms and conditions of the Creative Commons Attribution (CC BY) license (https:// creativecommons.org/licenses/by/ $4.0 /)$.

\begin{abstract}
The flexible and wearable capacitive sensors have captured tremendous interest due to their enormous potential for healthcare monitoring, soft robotics, and human-computer interface. However, despite recent progress, there are still pressing challenges to develop a fully integrated textile sensor array with good comfort, high sensitivity, multisensing capabilities, and ultra-light detection. Here, we demonstrate a pressure and non-contact bimodal fabric-only capacitive sensor with highly sensitive and ultralight detection. The graphene nanoplatelets-decorated multidimensional honeycomb fabric and nickel-plated woven fabric serve as the dielectric layer and electrode, respectively. Our textile-only capacitive bimodal sensor exhibits an excellent pressure-sensing sensitivity of $0.38 \mathrm{kPa}^{-1}$, an ultralow detection limit (1.23 Pa), and cycling stability. Moreover, the sensor exhibits superior non-contact detection performance with a detection distance of $15 \mathrm{~cm}$ and a maximum relative capacitance change of $10 \%$. The sensor can successfully detect human motion, such as finger bending, saliva swallowing, etc. Furthermore, a $4 \times 4$ (16 units) textile-only capacitive bimodal sensor array was prepared and has excellent spatial resolution and response performance, showing great potential for the wearable applications.
\end{abstract}

Keywords: wearable capacitive sensor; multifunctional; pressure; touchless; fabric

\section{Introduction}

Flexible and wearable electronic devices with various functionality and suitability for the human body have recently attracted considerable research interest with the rapid growth of artificial intelligence [1-3]. Flexible sensors, as a key element of wearable devices [4-6], have become one of the research hotspots due to their various promising applications [7,8], such as in human-machine interfaces, electronic skin, timely health monitoring, and soft robotics [9]. Based on the signal transition mechanisms, flexible sensors can be classified into capacitive [10,11], piezoelectric [12], resistive [13,14], and triboelectric types $[15,16]$. Among these, the capacitive sensors had been widely applied based on the advantages of outstanding temperature insensitivity, low power consumption, rapid dynamic response, and simple architecture design [17]. However, an urgent problem still exists for capacitive sensors to simultaneously achieve flexibility, wearability, comfort, and excellent multifunctional sensing capabilities (e.g., pressure, strain, temperature, etc.).

For improving the flexibility and wearability of the capacitive sensors, polymer elastomers, including polydimethylsiloxane (PDMS) [18], polyethylene terephthalate (PET) [19], polyimide (PI) [20], polyvinyl alcohol (PVA) [21], Eco-flex, and polyvinylidene fluoride (PVDF) [22,23], are often used to prepare the flexible electrodes and dielectric layers [24,25]. However, their pressure sensitivity performance still needs further improvement. Therefore, 
a simple method of incorporating conductive fillers into the dielectric layer of polymer elastomers to effectively improve their pressure sensitivity performance has been extensively studied $[26,27]$. Under applied pressure, the addition of a conductive filler could increase the dielectric constant, owing to the percolation threshold theory [28], which cause a change in capacitance [29]. Additionally, in the non-contact detection mode, the sensor can perceive and track the shape and position of the object without physical contact and realize the interaction with the surrounding environment, reflecting its unique advantages in the context of the COVID-19 pandemic. Zhang et al. [30] developed a stretchable dual-mode sensor array, exhibiting a $4 \%$ relative capacitance variation with a non-contact distance of $10 \mathrm{~cm}$ that could still be clearly detected. Sarwar et al. [17] reported a transparent tactile sensor based on a hydrogel electrode with an absolute value of $15 \%$ maximum capacitance relative change. However, limited by the structure and material characteristics of the traditional film-based or resin-based capacitive sensor, their poor air permeability is not conducive to sweat evaporation, which greatly hinders the long-term application of this capacitive sensor in the field of wearable electronics. Hence, flexible capacitive sensors with high breathability are still necessary for the improvement of comfort and durability.

For addressing these challenges, textile-based capacitive sensors, which could be divided into fiber-based [31] and fabric-based [32] capacitive sensors, have been reported to improve the permeability of flexible capacitive sensors due to their inherent nature of light weight, breathability, discretion, deformability, softness, and comfort [33-36]. Lee et al. fabricated a capacitive textile pressure sensor with a sensitivity of $0.21 \mathrm{kPa}^{-1}$ by coating PDMS on the surface of the conductive fiber as a dielectric layer and vertically stacking the two PDMS-coated fibers [37]. Chen et al. assembled textile-based capacitive sensors by electrospinning the electrode and nylon dielectric constant, which can accurately detect human joint motion [38]. Therefore, textile-based capacitive sensors are in favor to simultaneously achieve flexibility, wearability, comfort, and excellent multifunctional sensing capabilities, which is a very meaningful research direction in the field of flexible and wearable capacitive sensors. However, the corresponding work is rarely reported, especially for fabric-based capacitive sensors with multifunctional sensing. Thus, the potential of fabric-based capacitive sensors for the multifunctional signal sensing should be further developed.

Among various textile materials, the 3D honeycomb fabric consisting of two separate mesh knitted fabrics and a supporting yarn layer has excellent wearing comfort and compression [39,40], making it a potential material for flexible electronics [41]. Herein, we report a pressure and non-contact bimodal all-fabric-based capacitive (BAFC) sensor fabricated by a feasible and simple low-cost manufacturing method. The BAFC sensor is assembled by the $3 \mathrm{D}$ honeycomb fabric dielectric layer and the top and bottom conductive $\mathrm{Ni}$-plated woven electrodes, giving the sensor an excellent pressure-sensing sensitivity $\left(0.38 \mathrm{kPa}^{-1}\right)$ and ultralow detection limit (1.23 Pa). Additionally, this textile-based sensor has an outstanding non-contact detection performance with a detection distance of $15 \mathrm{~cm}$ and a maximum relative capacitance change of $10 \%$. Moreover, we demonstrate that our sensor is capable of detecting human motion, such as joint bending, saliva swallowing, etc. The low-cost, fabric-only capacitive bimodal sensor arrays have excellent spatial resolution and response performance, showing great potential for the wearable applications.

\section{Experiments}

\subsection{Materials and Characterization}

The multidimensional honeycomb fabric (thickness: $\sim 3 \mathrm{~mm}$, weight: $230 \mathrm{~g} / \mathrm{m}^{2}$ ) was provided by Shaoxing Yunfa Needle Textile Co., Ltd (Shaoxing, China). The honeycomb fabric has a three-dimensional structure, consisting of an upper honeycomb knitting structure, lower knitting coil structure, and intermediate support yarn (Figure S1a-c). The mesh size of the upper honeycomb knitting fabric is approximately $2 \times 2 \mathrm{~mm}$. The honeycomb fabric is prepared by a polyethylene terephthalate (PET) fiber, which is a kind of high molecular compound produced by esterification polycondensation of monoethylene glycol 
and terephthalic acid (Figure S1d). The nickel-plated fabric (thickness: $\sim 3 \mathrm{~mm}, \leq 0.05 \Omega / \mathrm{sq}$ ) was provided by Lutai Textile Co., P.R. (Zibo, China). The nickel-plated fabric substrate is woven from polyethylene terephthalate (PET) filaments, which have the same composition as honeycomb fabric. The graphene dispersion was supplied by Ningbo Morsh Technology.Co.,Ltd. (Ningbo, China).

The microscopic morphologies were characterized by field emission scanning electron microscopy (FESEM, REGULUS8100, Hitachi Production Institute of Zhuzhou Society, tokyo, Japan). The capacitance variation of the sensor was measured using a LCR digital bridge tester (ECA200A, Creek Valley Polymer Technology Co., Ltd, Beijing, China) at $200 \mathrm{kHz}$ frequency with a $1 \mathrm{~V}$ AC signal. The LCR digital bridge tester has a frequency range of $20 \mathrm{~Hz}$ to $200 \mathrm{kHz}$ and a basic accuracy of $0.1 \%$. An electronic universal testing machine (50N, ZQ-990B, Dongguan Smart Precision Instrument Co., Ltd, Dongguan, China) was used to apply pressure to the sensors. A computer was employed to record the compression distances, forces, and capacitances. The capacitance equation of the parallel-plate capacitor is defined as follows:

$$
C=\frac{\varepsilon_{r} S}{4 \pi \mathrm{k} d}
$$

where $d, \mathrm{~S}$, and $\varepsilon_{r}$ denote the distance between the top and the bottom electrodes, the electrode overlap area, and the dielectric constant, respectively. The increase of the capacitance of the BAFC sensor is the combined effect of $\varepsilon_{r}$ and $d$. The sensor electrode distance $\mathrm{d}$ decreases under pressure (Figure S2a). Moreover, the addition of GNPs give rise to an increase of $\varepsilon_{r}$ in the dielectric layer for the BAFC sensor. The effective dielectric constant can be defined according to the percolation theory model [42]:

$$
\varepsilon_{r}=\varepsilon_{D} /\left|\mathrm{f}_{\mathrm{c}}-\mathrm{f}\right|^{\mathrm{s}}
$$

where $\varepsilon_{D}$ is the conductivity of the dielectric phases, and $f_{c}$ and $f$ denote the percolation threshold and filling factor, respectively. The effective conduction path of the dielectric layer increases under compression, which is beneficial to improving the effective dielectric constant $\varepsilon_{r}$ [28] (Figure S2b).

The sensitivity $\left(S_{P r e}\right)$ of the sensor is calculated by differentiating the curve or depicting the tangent of the curve, which is defined as:

$$
S_{\text {Pre }}=\frac{\delta\left(\Delta C / \mathrm{C}_{0}\right)}{\delta \mathrm{P}}
$$

where $\Delta C$ denotes the capacitance change, $\mathrm{C}_{0}$ denotes the initial capacitance value, and $\mathrm{P}$ represents the applied external pressure.

In the non-contact mode, the capacitance is logarithmically related to the distance between the hand and the top electrode of the BATC sensor, corresponding to the model developed by Garbini:

$$
C=(4 \varepsilon / \mathrm{W} \ln (2 \mathrm{H} / h) / \pi
$$

where $\mathrm{W}$ and $\mathrm{H}$ stand for the width and height of the approaching conductor, $h$ is the distance between the bottom of the conductor and the top electrode, and $\varepsilon^{\prime}$ is a constant factor. In the non-contact mode, a capacitance $C_{F}$ is formed between the nickel-plated fabric electrode and the finger in the touchless mode [17], which is coupled with $C_{M}$ between the BAFC sensor (Figure S3). The fringing electric field of the bimodal sensor is disturbed and partially intercepted by the finger as the third electrode [33].

\subsection{Fabrication of BAFC Sensor Based on 3D Honeycomb Fabric}

The fabrication approach of the BAFC sensor is schematically illustrated in Figure 1. The commercial multidimensional honeycomb fabric (HF) and nickel-plated conductive fabrics can be tailored into arbitrary shapes and sizes for different applications. Here, the HF and nickel-plated conductive fabric were cut into a desirable size $\left(2 \times 2 \mathrm{~cm}^{2}\right)$ for convenient assembling by using a laser cutter (WE-6040). The HF was immersed into the 
graphene dispersion $(1 \mathrm{mg} / \mathrm{mL})$ and ultrasonically treated for $10 \mathrm{~min}$ to make the graphene uniformly deposited on the fabric, followed by complete drying at $80{ }^{\circ} \mathrm{C}$ for about $50 \mathrm{~min}$. Then, the nickel-plated conductive fabric was fixed on the GNPs-decorated 3D honeycomb fabric (GHF) dielectric layer to assemble the BAFC sensor.

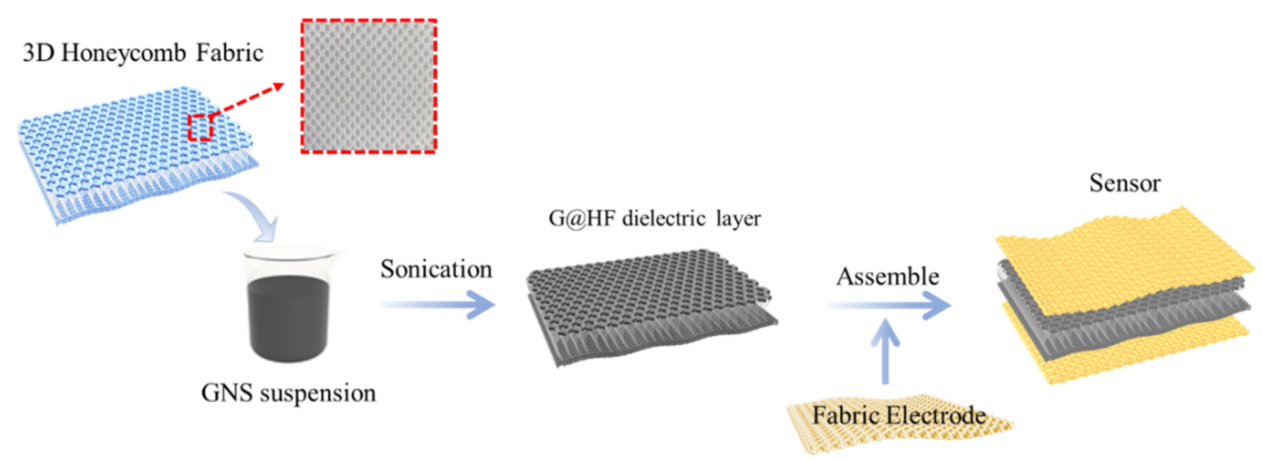

Figure 1. Schematical illustration of the preparation procedure for the BAFC sensor.

\section{Results and Discussion}

Figure 2 illustrates photographs and the typical surface morphology of the GHF dielectric layer and nickel-plating fabric electrode. As shown in Figure $2 \mathrm{a}-\mathrm{c}$, the GHF dielectric layer is constructed by an upper honeycomb knitted structure, lower knitted coil structure, and intermediate support yarns, giving fabric excellent resilience, softness, air permeability, and moisture permeability. The honeycomb and stereoscopic structure are particularly conductive to the free flow of air and water, forming a microcirculation air layer. Furthermore, the supporting yarns and lower compact knitted structure provide outstanding mechanical properties. The enlarged images in Figure $2 b, c$ showed that GNPs were successfully deposited on the surface of the HF fibers without agglomeration, which was beneficial to the improvement of the effective dielectric constant. The nickel-plating fabric is a plain weave structure, exhibiting the advantages of a smooth surface, strong texture, lightweight, and superior permeability (Figure 2d). Additionally, Figure 2e,f shows that nickel is uniformly wrapped on the fiber, offering the superior electrical conductivity.

The basic pressure-sensing properties of the BAFC sensor are quantitatively investigated in Figure 3. Figure $3 a$ exhibits the variation of capacitance in a wide pressure detection range of $0-120 \mathrm{kPa}$. The results show that the BAFC sensor has a high-pressure sensitivity of $0.038 \mathrm{kPa}^{-1}$. The BAFC sensor was tested in three successive load/unload cycles at each of three external pressures $(8,40$, and $90 \mathrm{kPa})$, demonstrating a stable and sequential capacitance response (Figure $3 b$ ). The ultralow detection limit of the bimodal G@HF-based capacitive sensor is worthy of concern. It was evaluated by using a small paper $(1.23 \mathrm{~Pa})$. The BAFC sensor can achieve relative capacitance changes $\left(\Delta C / \mathrm{C}_{0}\right)$ of $0.37 \%$, denoting the superior low pressure detection capability (Figure 3c). To further investigate the durability and stability of the sensor, the capacitance variations were recorded by applying repeated loading/unloading pressure $(6 \mathrm{kPa})$ over 2000 cycles (Figure 3d). The sensor retained a stable response with no significant drift. In addition, the spatial distinguishing ability of the sensor array is crucial to the field of artificial intelligence, such as human-computer interaction. Here, a $4 \times 4$ (16 units) BAFC sensor array was fabricated for the following experimental tests. In order to verify its perception ability of detecting spatial pressure distribution, a red bean $(0.72 \mathrm{~g})$ was placed on the array, corresponding to $\Delta \mathrm{C} / \mathrm{C}_{0}$ of $2.5 \%$ (Figure $3 \mathrm{e}$ ). This result demonstrates that the sensor array can achieve high-resolution pressure mapping and object localization. To explore wearable applications in dynamic response and human motion recognition, relevant preliminary research tests were conducted. The sensor was fixed on the finger joint, and the relationship between the capacitance variations and finger bending angles is displayed in Figure 3f,g. The capacitance of the BAFC sensor increases with the increase of the finger bending angle. The BAFC sensor was attached to the surface of the wrist to measure its sensing performance. As 
shown in Figure 3h, the BAFC sensor exhibits a repeatable and reliable sensing performance under repetitive bending/relaxation motions.
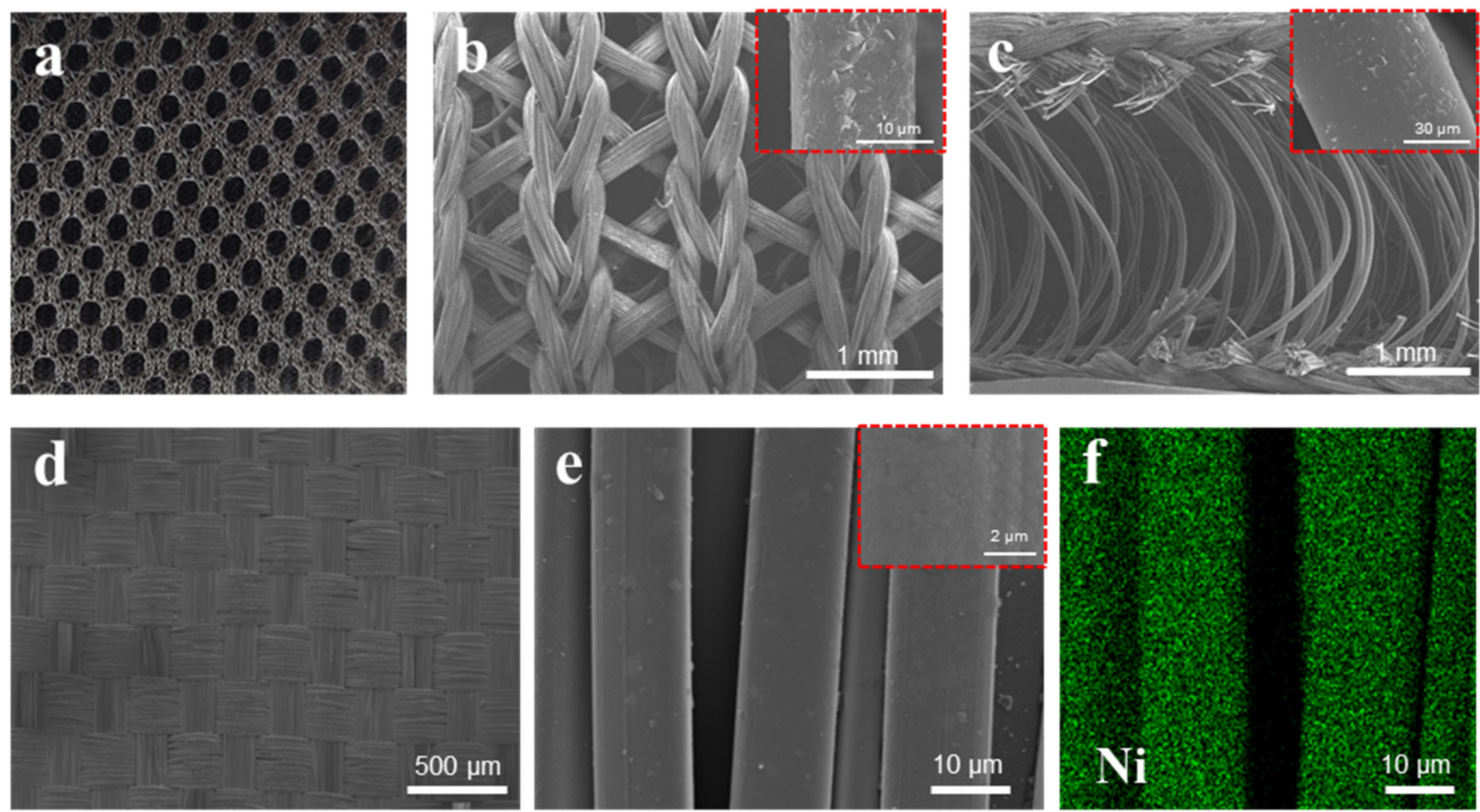

Figure 2. Photographs of the typical surface morphology: (a) upper honeycomb structure images of the GHF dielectric layer; FESEM images of (b) the lower compact knitted structure and (c) the supporting yarn of the GHF; (d,e) the woven structure; (f) energy dispersive spectroscopy (EDS) mapping of the nickel-plating fabric electrode.

The performance of the BAFC sensor for proximity monitoring is displayed in Figure 4 . A hand vertically approached the sensor to determine the touchless detection capability of the BAFC sensor. As can be observed from Figure $4 \mathrm{a}$, the bimodal sensor has a large detection range $(0-15 \mathrm{~cm})$, with a relative capacitance variation of up to $10 \%$. Furthermore, a maximum sensitivity of $0.023 \mathrm{~cm}^{-1}$ is computed for the low distance range $(<2 \mathrm{~cm})$. Moreover, Figure $4 \mathrm{~b}$ shows that the capacitance variations are steadily maintained when a hand is hovered at different distances, demonstrating a steady non-contact sensing response of the BAFC sensor. The BAFC sensor can successfully transmit information or emergency signals through the Morse code (Figure 4c). Furthermore, Figure 4d,e shows that the capacitance change rate of the corresponding sensor unit is $10 \%$ when the finger is suspended above the 42 sensor array unit. This proves that the BAFC sensor array can accurately locate the finger and has an excellent non-contact spatial response performance.

The BAFC sensor was evaluated to detect subtle pressure, such as weak airflow, swallowing, blinking, etc. Mechanical bending and vibration detection (contact-type detection) can be used to detect human physiological signal, and airflow detection (non-contact pressure detection) can be used to detect the direction and intensity of the airflow. Therefore, both non-contact pressure sensing and contact pressure sensing have important research significance in the wearable field. Furthermore, airflow detection is widely needed in meteorological monitoring, biomedical engineering, aerospace, and mining enterprises [43]. However, non-contact pressure detection property of sensors is often overlooked. Therefore, the airflow sensation, one of the important human perception functions [44], was also evaluated. Figure 5 a shows that the sensor can sense and convert pressure mechanical signals generated by airflow from the blower into electrical signals. Figure $5 b, c$ present the capacitance response of the BAFC sensor attached to the throat during saliva swallowing. 
The various peak intensities are determined by the different extents of the vibration of the intrinsic laryngeal muscles. Similarly, the sensor was attached to the forehead to monitor the human facial muscles changes. As shown in Figure 5d, when the volunteers frowned, the BAFC sensor clearly showed the growth rate of a $1-1.5 \%$ relative capacitance change. Moreover, the capacitance of the sensor returned to the initial value when volunteers relaxed their eyebrows. This proves that the sensor promptly sensed the occurrence of frown movements. Additionally, the BAFC sensor was also applied to detect eye muscle movement (Figure 5e). The BAFC sensor showed a growth rate of a $0.6 \%$ relative capacitance change with volunteers' eyes closed, which showed that it accurately captured the compressive stress generated by the opening/closing movement of the eyes. The repetitive capacitance change signal caused by repeated blink movement confirmed that the BAFC sensor could be well used to detect abnormal convulsion caused by eye diseases. These results demonstrate the BAFC can identify human facial expression changes, exhibiting enormous potentiality in wearable flexible electronics.

a
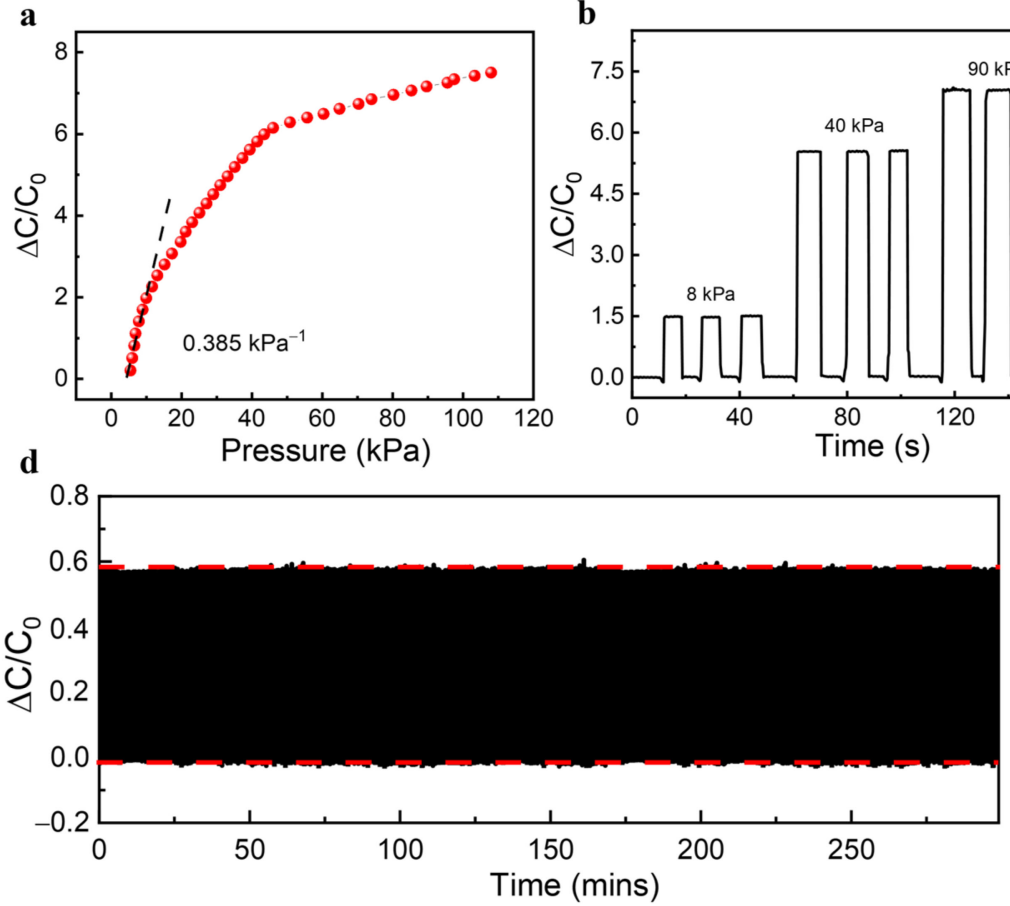

b

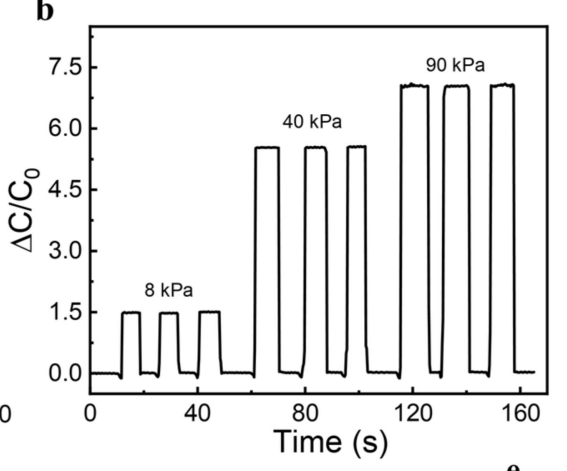

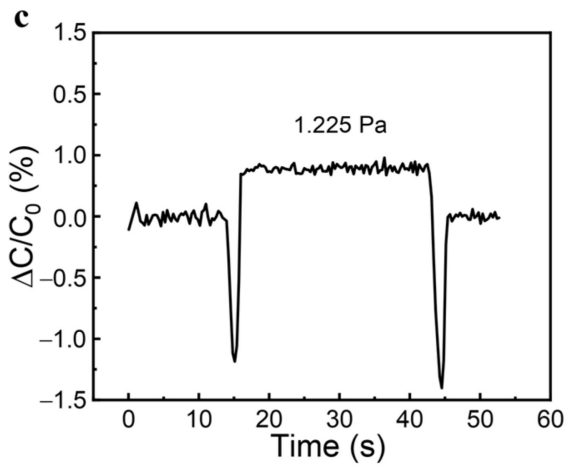

f

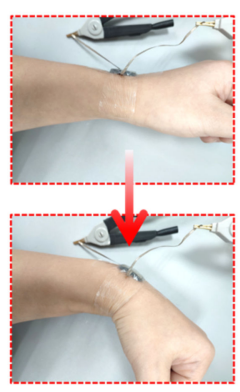

g

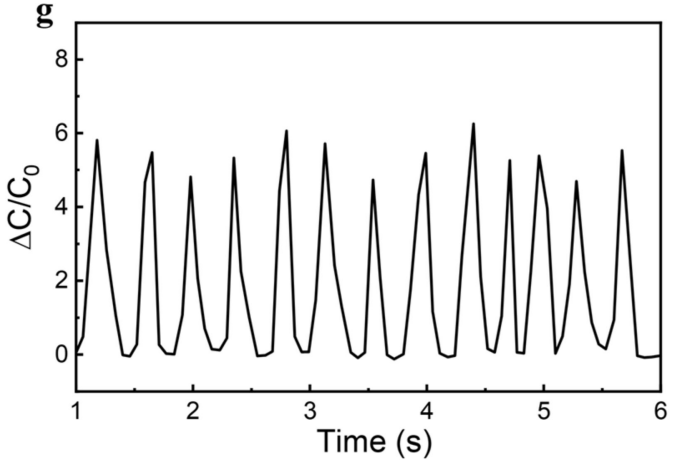

h

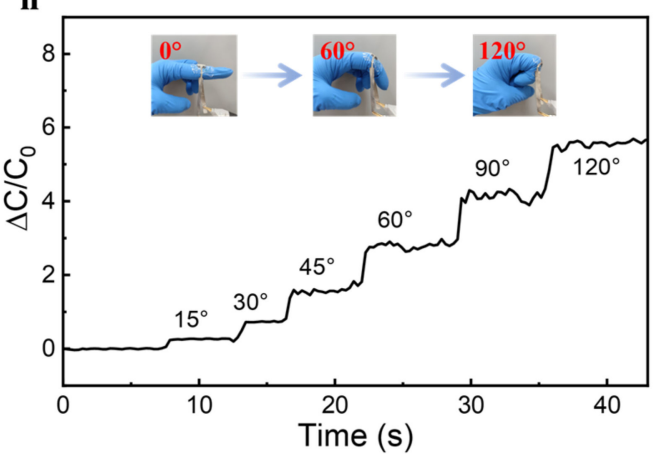

Figure 3. Pressure-sensing performance of the BAFC sensor: (a) relative capacitance response and sensitivity of the BAFC sensor; (b) capacitive response of the sensor for different loads of 8, 40, and $90 \mathrm{kPa}$; (c) ultra-light pressure responses; (d) durability of the capacitance response of the BAFC sensor to 2000 cycles under a loading pressure of $6 \mathrm{kPa}$; (e) pressure spatial mapping of a red bean; $(\mathbf{f}, \mathbf{g})$ capacitance change of the BAFC sensor according to wrist bending motion; (h) representative capacitance signal during the bending and unbending of finger to angles of $15,30,45,60,90$, and $120^{\circ}$. 

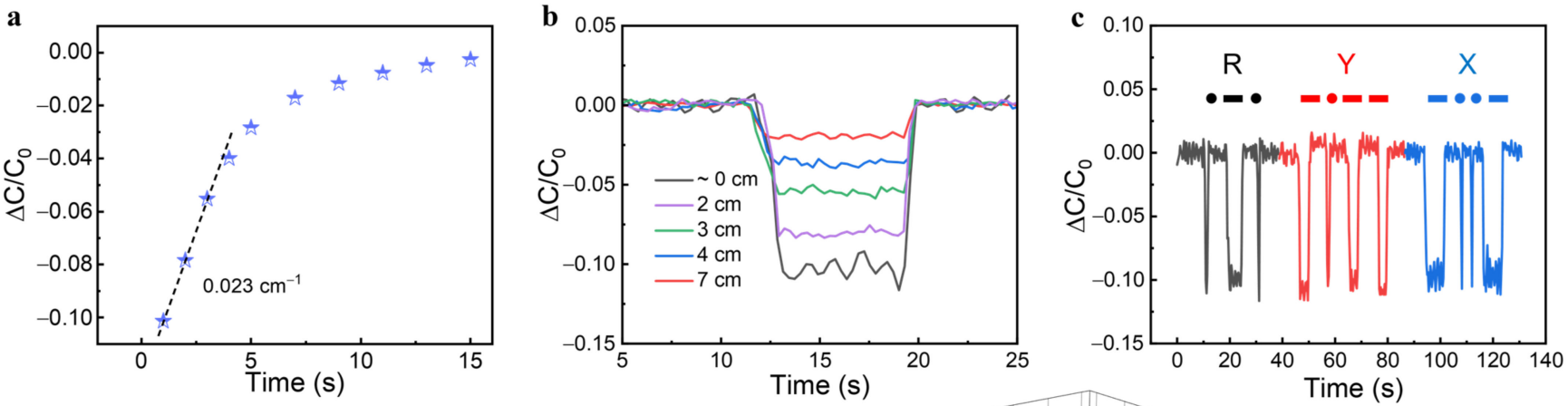

d

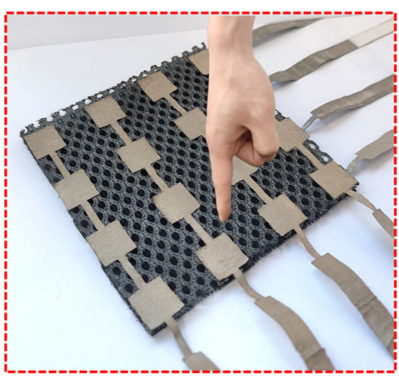

e

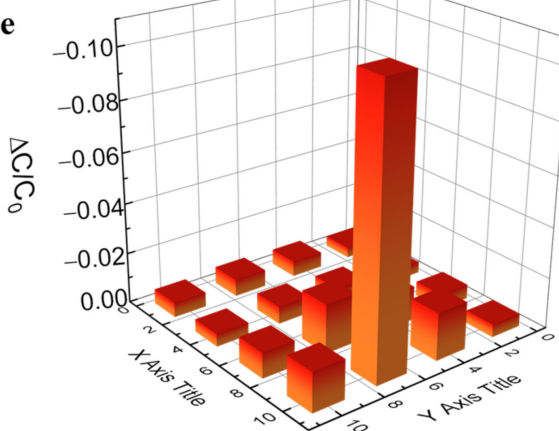

Figure 4. Non-contact sensing performance of the BAFC sensor: (a) relative capacitance change responding to the approaching hand; (b) static capacitance response at different distances between the hand and the sensor array; (c) international Morse password signal; (d,e) non-contact functionality of the BAFC sensor for the positioning finger.

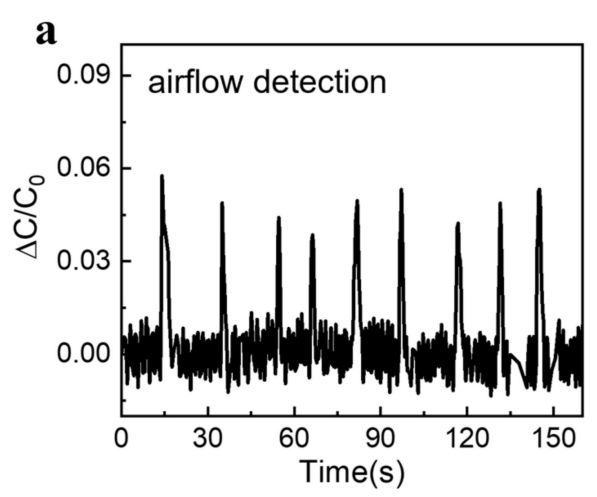

d

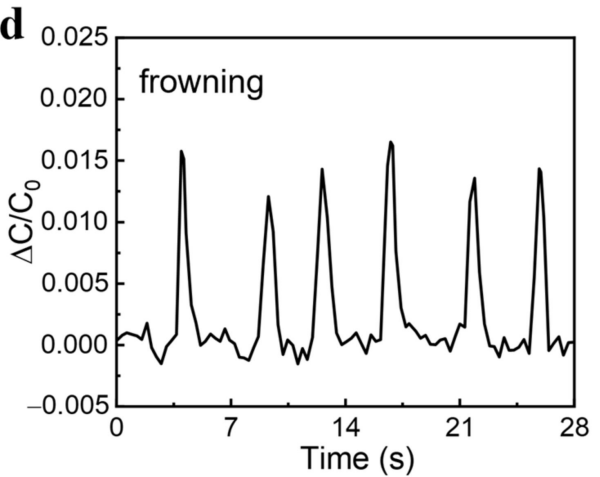

b

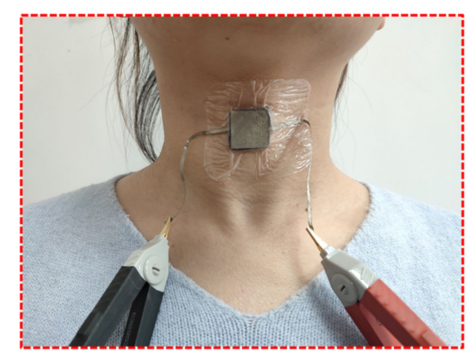

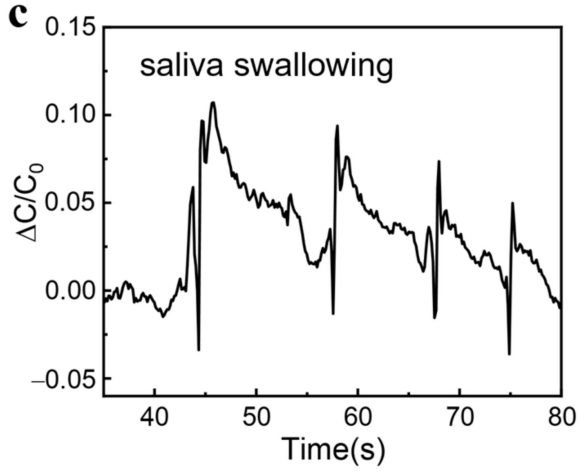

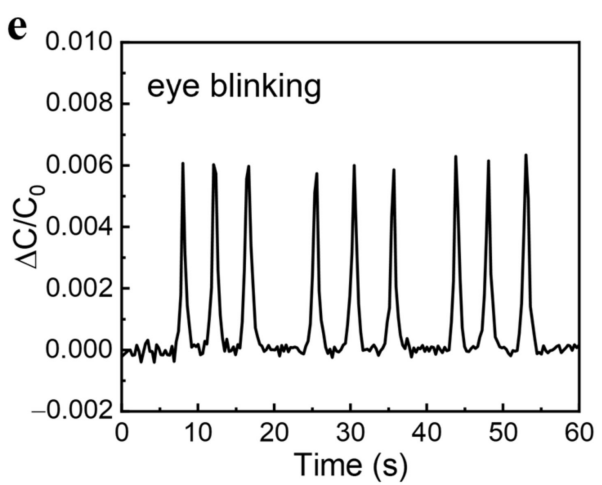

Figure 5. The micropressure monitoring of the BAFC sensor: (a) electrical signal of air flow detection; (b) photographs of the sensor attached to detect muscle movement during saliva swallowing. Timedependent capacitance responses for the corresponding (c) saliva swallowing, (d) frowning, and (e) eye blinking movements. 


\section{Conclusions}

In summary, this paper has reported a bimodal all-fabric-based capacitive sensor with a non-contact and pressure stimulus response via a feasible and simple low-cost manufacturing method. This all-fabric-based construction, constructed by the 3D honeycomb knitted fabric dielectric layer and nickel-plated plain-woven fabric electrode, endows the sensor with superior breathability, moisture permeability, and mechanical stability. The GHF-based bimodal sensor can successfully monitor dual non-contact and pressure-sensing responses. Moreover, it has an excellent pressure-sensing sensitivity $\left(0.38 \mathrm{kPa}^{-1}\right)$ and noncontact detection performance (detection distance: $15 \mathrm{~cm}$, maximum relative capacitance change: $10 \%$ ). This bimodal wearable sensor also exhibits an ultralow detection limit $(1.23 \mathrm{~Pa})$ and cycling stability $(>2000)$. In addition, the BAFC sensor array can achieve high-resolution pressure and non-contact mapping and object localization. Furthermore, our BAFC sensor can accurately monitor the human motion and physiological signals in real-time, exhibiting great potential in wearable, flexible electronics.

Supplementary Materials: The following are available online at https:/ /www.mdpi.com/article/10 .3390 / coatings12030302/s1, Figure S1. (a) Upper honeycomb structure, (b) lower knitted structure and (c) support yarns FESEM images of the HF. Figure S2. The mechanism diagram of pressure sensing. (a) The variation of electrode distance (d) under external pressure. (b) Schematic illustration of the hybrid conductive network constructed by GNPs. Figure S3. The mechanism diagram of non-contact sensing.

Author Contributions: Conceptualization and supervision, H.W. and M.T.; investigation and writing, X.Y.; resources and validation, M.L. and Y.S. All authors have read and agreed to the published version of the manuscript.

Funding: This research was funded by the Shandong Province Key Research and Development Plan (2019JZZY010340, 2019JZZY010335), Anhui Province Special Science and Technology Project (201903a05020028), Shandong Provincial Universities Youth Innovation Technology Plan Team (2020KJA013), Natural Science Foundation of Shandong Province (ZR2020QE074), and Nantong Science and Technology Plan Project (JCZ18058).

Institutional Review Board Statement: Not applicable.

Informed Consent Statement: Not applicable.

Data Availability Statement: Not applicable.

Acknowledgments: We want to thank for the support received from the Shandong Province Key Research and Development Plan (2019JZZY010340, 2019JZZY010335), Anhui Province Special Science and Technology Project (201903a05020028), Shandong Provincial Universities Youth Innovation Technology Plan Team (2020KJA013), Natural Science Foundation of Shandong Province (ZR2020QE074), and Nantong Science and Technology Plan Project (JCZ18058). Furthermore, we thank Weidong Zhang from Jiangsu College of Engineering and Technology for some supports in testing and data analysis.

Conflicts of Interest: The authors declare no conflict of interest.

\section{References}

1. Shi, J.; Liu, S.; Zhang, L.; Yang, B.; Shu, L.; Yang, Y.; Ren, M.; Wang, Y.; Chen, J.; Chen, W.; et al. Smart Textile-Integrated Microelectronic Systems for Wearable Applications. Adv. Mater. 2020, 32, e1901958. [CrossRef]

2. Dong, K.; Deng, J.; Ding, W.; Wang, A.C.; Wang, P.; Cheng, C.; Wang, Y.-C.; Jin, L.; Gu, B.; Sun, B.; et al. Versatile Core-Sheath Yarn for Sustainable Biomechanical Energy Harvesting and Real-Time Human-Interactive Sensing. Adv. Energy Mater. 2018, 8, 1801114. [CrossRef]

3. He, F.; You, X.; Wang, W.; Bai, T.; Xue, G.; Ye, M. Recent Progress in Flexible Microstructural Pressure Sensors toward HumanMachine Interaction and Healthcare Applications. Small Methods 2021, 5, 2001041. [CrossRef]

4. Li, Y.; Zhang, M.; Hu, X.; Yu, L.; Fan, X. Graphdiyne-based flexible respiration sensors for monitoring human health. Nano Today 2021, 39, 101214. [CrossRef]

5. Yang, X.; Wang, Y.; Qing, X. Electrospun Ionic Nanofiber Membrane-Based Fast and Highly Sensitive Capacitive Pressure Sensor. IEEE Access 2019, 7, 139984-139993. [CrossRef] 
6. Li, S.; Li, Y.; Liu, X.; Li, X.; Ding, T.; Ouyang, H. An In-Situ Electroplating Fabricated Fabry-Perot Interferometric Sensor and Its Temperature Sensing Characteristics. Coatings 2020, 10, 1174. [CrossRef]

7. Dong, K.; Peng, X.; An, J.; Wang, A.C.; Luo, J.; Sun, B.; Wang, J.; Wang, Z.L. Shape adaptable and highly resilient 3D braided triboelectric nanogenerators as e-textiles for power and sensing. Nat. Commun. 2020, 11, 2868. [CrossRef] [PubMed]

8. Li, M.; Li, Z.; Ye, X.; Zhang, X.; Qu, L.; Tian, M. Tendril-Inspired 900\% Ultrastretching Fiber-Based Zn-Ion Batteries for Wearable Energy Textiles. ACS Appl. Mater. Inter. 2021, 13, 17110-17117. [CrossRef]

9. Luo, C.; Liu, X.; Liu, J.; Shen, J.; Li, H.; Zhang, S.; Hu, J.; Zhang, Q.; Wang, G.; Huang, M. An Optimized PDMS Thin Film Immersed Fabry-Perot Fiber Optic Pressure Sensor for Sensitivity Enhancement. Coatings 2019, 9, 290. [CrossRef]

10. Ma, Y.; Ouyang, J.; Raza, T.; Li, P.; Jian, A.; Li, Z.; Liu, H.; Chen, M.; Zhang, X.; Qu, L.; et al. Flexible all-textile dual tactile-tension sensors for monitoring athletic motion during taekwondo. Nano Energy 2021, 85, 105941. [CrossRef]

11. Gao, L.; Wang, M.; Wang, W.; Xu, H. Highly Sensitive Pseudocapacitive Iontronic Pressure Sensor with Broad Sensing Range. Nano-Micro Lett. 2021, 13, 140. [CrossRef]

12. He, S.; Guo, M.; Dan, Z. Large-area atomic-smooth polyvinylidene fluoride Langmuir-Blodgett film exhibiting significantly improved ferroelectric and piezoelectric responses. Sci. Bull. 2021, 66, 1080-1090. [CrossRef]

13. Yin, X.-Y.; Zhang, Y.; Cai, X.; Guo, Q.; Yang, J.; Wang, Z.L. 3D printing of ionic conductors for high-sensitivity wearable sensors. Mater. Horiz. 2019, 6, 767-780. [CrossRef]

14. Guo, H.; Tan, Y.J.; Chen, G.; Wang, Z.; Susanto, G.J. Artificially innervated self-healing foams as synthetic piezo-impedance sensor skins. Nat. Commun. 2020, 11, 5747. [CrossRef]

15. Dong, K.; Deng, J.; Zi, Y.; Wang, Y.-C.; Xu, C.; Zou, H.; Ding, W.; Dai, Y.; Gu, B.; Sun, B.; et al. 3D Orthogonal Woven Triboelectric Nanogenerator for Effective Biomechanical Energy Harvesting and as Self-Powered Active Motion Sensors. Adv. Mater. 2017, 29, 1702648. [CrossRef] [PubMed]

16. Dong, K.; Wang, Z.L. Self-charging power textiles integrating energy harvesting triboelectric nanogenerators with energy storage batteries/supercapacitors. J. Semicon. 2021, 42, 101601. [CrossRef]

17. Sarwar, M.; Dobashi, Y.; Preston, C.; Wyss, J.; Mirabbasi, S.; Madden, J. Bend, stretch, and touch: Locating a finger on an actively deformed transparent sensor array. Sci. Adv. 2017, 3, e1602200. [CrossRef]

18. Tay, R.Y.; Li, H.; Lin, J.; Wang, H.; Lim, J.S.K.; Chen, S.; Leong, W.L.; Tsang, S.H.; Teo, E.H.T. Lightweight, Superelastic Boron Nitride/Polydimethylsiloxane Foam as Air Dielectric Substitute for Multifunctional Capacitive Sensor Applications. Adv. Funct. Mater. 2020, 30, 223. [CrossRef]

19. Kang, M.; Kim, J.; Jang, B.; Chae, Y.; Kim, J.H.; Ahn, J.H. Graphene-Based Three-Dimensional Capacitive Touch Sensor for Wearable Electronics. ACS Nano 2017, 11, 7950-7957. [CrossRef]

20. Zhu, Y.; Wu, Y.; Wang, G.; Wang, Z.; Tan, Q.; Zhao, L.; Wu, D. A flexible capacitive pressure sensor based on an electrospun polyimide nanofiber membrane. Org. Electron. 2020, 84, 105759. [CrossRef]

21. Ding, W.; Lu, L.; Chen, Y.; Liu, J.; Yang, B. Flexible P(VDF-TrFE) Shared Bottom Electrode Sensor Array Assisted with Machine Learning for Motion Detection. Coatings 2020, 10, 1094. [CrossRef]

22. Shi, H.; Al-Rubaiai, M.; Holbrook, C.M.; Miao, J.; Pinto, T.; Wang, C.; Tan, X. Screen-Printed Soft Capacitive Sensors for Spatial Mapping of Both Positive and Negative Pressures. Adv. Funct. Mater. 2019, 29, 1903020. [CrossRef]

23. Pyo, S.; Choi, J.; Kim, J. Flexible, Transparent, Sensitive, and Crosstalk-Free Capacitive Tactile Sensor Array Based on Graphene Electrodes and Air Dielectric. Adv. Energy Mater. 2018, 4, 1700427. [CrossRef]

24. Zhang, J.; Wan, L.; Gao, Y.; Fang, X.; Lu, T.; Pan, L.; Xuan, F. Highly Stretchable and Self-Healable MXene/Polyvinyl Alcohol Hydrogel Electrode for Wearable Capacitive Electronic Skin. Adv. Energy Mater. 2019, 5, 1900285. [CrossRef]

25. Zhou, H.; Wang, Z.; Zhao, W.; Tong, X.; Jin, X.; Zhang, X.; Yu, Y.; Liu, H.; Ma, Y.; Li, S.; et al. Robust and sensitive pressure/strain sensors from solution processable composite hydrogels enhanced by hollow-structured conducting polymers. Chem. Eng. J. 2021, 403, 126307. [CrossRef]

26. Ke, K.; McMaster, M.; Christopherson, W.; Singer, K.D.; Manas-Zloczower, I. Highly sensitive capacitive pressure sensors based on elastomer composites with carbon filler hybrids. Compo. Part A-Appl. Sci. Manuf. 2019, 126, 105614. [CrossRef]

27. Chen, L.; Chen, G.H.; Lu, L. Piezoresistive Behavior Study on Finger-Sensing Silicone Rubber/Graphite Nanosheet Nanocomposites. Adv. Funct. Mater. 2007, 17, 898-904. [CrossRef]

28. Qiu, J.; Guo, X.; Chu, R.; Wang, S.; Zeng, W.; Qu, L.; Zhao, Y.; Yan, F.; Xing, G. Rapid-Response, Low Detection Limit, and High-Sensitivity Capacitive Flexible Tactile Sensor Based on Three-Dimensional Porous Dielectric Layer for Wearable Electronic Skin. ACS Appl Mater. Inter. 2019, 11, 40716-40725. [CrossRef]

29. Moheimani, R.; Aliahmad, N.; Aliheidari, N.; Agarwal, M.; Dalir, H. Thermoplastic polyurethane flexible capacitive proximity sensor reinforced by CNTs for applications in the creative industries. Sci. Rep. 2021, 11, 1104. [CrossRef]

30. Zhang, C.; Liu, S.; Huang, X.; Guo, W.; Li, Y.; Wu, H. A stretchable dual-mode sensor array for multifunctional robotic electronic skin. Nano Energy 2019, 62, 164-170. [CrossRef]

31. Keum, K.; Eom, J.; Lee, J.H.; Heo, J.S.; Park, S.K.; Kim, Y.-H. Fully-integrated wearable pressure sensor array enabled by highly sensitive textile-based capacitive ionotronic devices. Nano Energy 2021, 79, 105479. [CrossRef]

32. Wu, R.; Ma, L.; Patil, A.; Hou, C.; Zhu, S.; Fan, X.; Lin, H.; Yu, W.; Guo, W.; Liu, X.Y. All-Textile Electronic Skin Enabled by Highly Elastic Spacer Fabric and Conductive Fibers. ACS Appl. Mater. Inter. 2019, 11, 33336-33346. [CrossRef] 
33. Guan, F.; Xie, Y.; Wu, H.; Meng, Y.; Shi, Y.; Gao, M.; Zhang, Z.; Chen, S.; Chen, Y.; Wang, H.; et al. Silver Nanowire-Bacterial Cellulose Composite Fiber-Based Sensor for Highly Sensitive Detection of Pressure and Proximity. ACS Nano 2020, 14, 15428-15439. [CrossRef]

34. Wang, H.; Li, X.; Zhuang, X.; Cheng, B.; Wang, W.; Kang, W.; Shi, L.; Li, H. Modification of Nafion membrane with biofunctional $\mathrm{SiO} 2$ nanofiber for proton exchange membrane fuel cells. J. Power Sources 2017, 340, 201-209. [CrossRef]

35. Dong, K.; Hu, Y.; Yang, J.; Kim, S.-W.; Hu, W.; Wang, Z.L. Smart textile triboelectric nanogenerators: Current status and perspectives. MRS Bull. 2021, 46, 512-521. [CrossRef]

36. Wang, L.; Wang, L.; Zhang, Y.; Pan, J.; Li, S.; Sun, X.; Zhang, B.; Peng, H. Weaving Sensing Fibers into Electrochemical Fabric for Real-Time Health Monitoring. Adv. Funct. Mater. 2018, 28, 1804456. [CrossRef]

37. Lee, J.; Kwon, H.; Seo, J.; Shin, S.; Koo, J.H.; Pang, C.; Son, S.; Kim, J.H.; Jang, Y.H.; Kim, D.E.; et al. Conductive fiber-based ultrasensitive textile pressure sensor for wearable electronics. Adv. Mater. 2015, 27, 2433-2439. [CrossRef] [PubMed]

38. Chen, Y.; Wang, Z.; Xu, R.; Wang, W.; Yu, D. A highly sensitive and wearable pressure sensor based on conductive polyacrylonitrile nanofibrous membrane via electroless silver plating. Chem. Eng. J. 2020, 394, 124960. [CrossRef]

39. Mousavi, G.; Varsei, M.; Rashidi, A.; Ghazisaeidi, R. Experimental evaluation of the compression garment produced from elastic spacer fabrics through real human limb. J. Ind. Text. 2021, 1528083720988089. [CrossRef]

40. Chen, C.Y.; Du, Z.Q.; Yu, W.D.; Dias, T. Analysis of physical properties and structure design of weft-knitted spacer fabric with high porosity. Text. Res. J. 2018, 88, 59-68. [CrossRef]

41. Wang, H.; Wang, X.; Fan, T.; Zhou, R.; Li, J.; Long, Y.; Zhuang, X.; Cheng, B. Fabrication of electrospun sulfonated poly(ether sulfone) nanofibers with amino modified $\mathrm{SiO} 2$ nanosphere for optimization of nanochannels in proton exchange membrane. Solid State Ion. 2020, 349, 115300. [CrossRef]

42. Pecharroman, C.; Moya, J.S. Experimental evidence of a giant capacitance in insulator-conductor composites at the percolation threshold. Adv. Mater. 2000, 12, 294-297. [CrossRef]

43. Wang, H.; Li, S.; Wang, Y.; Wang, H.; Shen, X.; Zhang, M.; Lu, H.; He, M.; Zhang, Y. Bioinspired Fluffy Fabric with In Situ Grown Carbon Nanotubes for Ultrasensitive Wearable Airflow Sensor. Adv. Mater. 2020, 32, e1908214. [CrossRef] [PubMed]

44. Huang, S.; Zhang, B.; Lin, Y.; Lee, C.S.; Zhang, X. Compact Biomimetic Hair Sensors Based on Single Silicon Nanowires for Ultrafast and Highly-Sensitive Airflow Detection. Nano Lett. 2021, 21, 4684-4691. [CrossRef] 